\title{
Influence of Flow Conditions in Entertainment Activities:
}

\section{Is Self-reported Levels of Flow State Higher in Video Games Than in TV Shows for 18-24 U.S. Undergraduate Students}

\author{
Jiayue $\mathrm{GaO}^{1, \mathrm{a}, *, \dagger}$, Zihan $\mathrm{Lu}^{2, \mathrm{~b},{ }^{*}, \dagger}$
}

\author{
${ }^{1}$ Irvine Valley College, California, U.S. \\ ${ }^{2}$ University of California, Davis, California, U.S. \\ *Corresponding author. Email: ${ }^{a}$ Cgao5@ivc.edu, ${ }^{b} z z z l u @ u c d a v i s . e d u$
}

These authors contributed equally.

\begin{abstract}
Flow is a subjective statement when an individual is completely engrossed in the activity and feels highly concentrated, involved and enjoyed. It will be of great benefit for undergraduate students if it can be applied to the academic field and skills training. Studying how to flow condition affects flow state scale in entertainment activities can help understand how the flow works in the entertainment field. This study conducted an online survey to test the hypothesis that flow condition and flow state scale have significant differences between video games and TV shows. The data showed a significant difference in flow condition between the two activities, but there is no significant difference in flow state scale between those two. This implies that flow conditions barely have an influence on flow state scale in entertainment activities. However, this may be due to other factors of TV shows which can promote levels of concentration, involvement, and enjoyment in different ways, such as the audience being passively entertained and focused because of its sensory stimuli, sufficient new knowledge, and intriguing storyline and characters.
\end{abstract}

Keywords: Flow, Entertainment, Concentration, Involvement, Enjoyment

\section{INTRODUCTION}

\subsection{The Concept of Flow}

Mihaly Csikszentmihaly, who was best known for establishing the concept of flow, came up with this notion when trying to figure out why artists, particularly painters, become so immersed in their artwork that they neglect their basic physical demands. According to Csikszentmihaly, flow is a subjective state when people self-reported that they are "completely involved in something to the point of forgetting time, fatigue, and everything else but the activity itself" [1]. Understanding how flow works can help individuals foster the ability to embrace challenges and conquer them, which is of great importance from personal and cultural perspectives.

\subsection{The Importance of Flow for College Students}

When someone is in a flow state, they are completely focused on the task at hand and remain dedicated to it. Understanding how the flow works is quite beneficial for college students to become more productive in their academic and personal lives. Students who comprehend how flow works may use it in the learning activities to increase productivity and achieve personal goals. Furthermore, appropriate stress management is essential for college students to maintain mental wellness. When dealing with stress and strain, entertainment activities and workouts might be beneficial. As a result, integrating the principle of flow with practice in leisure activities and exercise may be quite beneficial in stress reduction. 


\subsection{The Role of Video Games and TV Shows among College Students}

The advancement of technology in the 21 st century leads to a longer time spent on online activities among college students within entertainment. Specifically, individuals spend most of their time on social media such as Instagram, Facebook, Twitter, and other e-gaming activities. Within the percentage of the population who plays video games, studies show that college students account for a large proportion simply because of the lack of parental supervision as external pressure and the flexibility of the class schedule [2]. Another popular digital entertainment is Television shows that attract a long attention span from college students by delivering online streaming. Based on a purposive survey, college students who are heavy users of the online internet, including watching TV shows tend to spend more time online checking emails, downloading files, and use online messaging tools [3].

\section{LITERATURE REVIEW}

\subsection{Flow in Entertainment}

The concepts associated with the occurrence of flow can be implied by playing video games and watching television shows in entertainment. The main goal of both activities is to let the participant fully immerse in the zone through intrinsic motivation, which results in the sense of absence of time perception. Csikszentmihalyi's theory states that balancing internal skills and external challenges while performing tasks is the key to the Flow experience. With that being said, a gain in abilities is the result of learning, and novelty increases the difficulty of performing a task [4].

The use of levels in games, for example, provides small sections that lead to the completion of the entire task, which increases the level of concentration due to the balance of one's own ability and the difficulty of the game levels [4]. On top of a fairly challenging game setup, the other two conditions, including a clear set of goals and immediate feedback, also increase the players' attention span on particular tasks. The small tasks contained within the games, such as collecting points, gathering objects and artifacts, solving the puzzle...etc., provide a direct purpose for the player to behave a certain way to achieve the goal. On the other hand, immediate feedback corresponds with the player's action, which provides instant response. To support the point, researchers conclude that "shooting someone and they die" or "find a clue, and you can put it in your bag" are some of the manifestations in games that are part of the element to reach flow state [4].

To compare and contrast, an individual watching television shows doesn't experience challenges of skill set or have a clear purpose other than entertaining.
However, the sensory and auditory stimuli did play an important role in contributing to the flow stage. On the other hand, the stage of development attracts the audience's attention by creating dramatic rising action and climax, which increase attention span on a particular scene. The higher concentration someone has on the show, the more likely they will enter a flow state [3].

\subsection{The Conditions of Flow}

Flow experience can occur when certain conditions are met. Previous research has studied the conditions for flow to exist, and Csikszentmihalyi and his colleagues concluded three critical conditions [1]. The first precondition for flow experience is a set of clear goals. These goals can guide participants by adding direction and purpose to the activities. A second condition for flow to exist is the optimal challenge. That means the difficulty of the behavior is well-matched with the ability of the participants. Since overwhelming challenges can typically cause anxiety, conversely, one can be relaxed and bored when the action is oversimplified. Thirdly, flow experience tends to happen when clear and immediate feedback is presented. Clear and immediate feedback can strengthen the certainty for participants of how well they are dealing with the current level of challenges and provide instructions on what to do next.

\subsection{The Measurements of Flow}

Since the flow is subjective, the flow measurements are usually completed by the experience sampling method (ESM), self-reported questionnaire, and interview. Researchers have developed many self-report instruments for assessing flow in different kinds of activities and fields. The flow state scale-2 (FSS-2) and the dispositional flow scale-2 (DFS-2) developed by Jackson and his colleagues have been acknowledged as the standardized scales that are most commonly used [5, 6]. FSS-2 is used to measure flow experience in physical activities, and DFS-2 measures the tendency to experience flow in particular domains, which is also validated among internet gamers [7]. Each scale has an original long version and a modified short version, and all of them use a 5-point Likert scale to measure the nine dimensions of flow [8].

\subsection{Value of the Study}

Recent studies have made an in-depth analysis of the way to access flow state and the experience of flow in different kinds of activities. Most studies have focused on physical activities and academic activities. Though flow has been widely discussed when developing video games, few studies show that flow condition significantly influences entertainment activities on the flow state level. This study makes a comparison between video games (an entertainment activity that fulfills the flow conditions theoretically) and TV shows (an 
entertainment activity that does not meet the flow condition theoretically) to investigate, at the statistical level, whether there is a significant difference in flow state scale between the two activities. This study's survey design and method are mainly based on the theory of flow conditions and flow measurement mentioned above.

\section{METHODOLOGY}

\subsection{Participants}

To test the hypothesis, an online survey is conducted. It aims to learn about self-reported levels of flow conditions, concentration, involvement, and enjoyment for video games (an entertainment activity that is supposed to fulfill the flow conditions) and TV shows (an entertainment activity that is supposed to do not meet the flow condition). This survey was distributed through an online tool, QuestionPro. Participants were randomly selected teenagers users of QuestionPro between 18-24 years old. This survey received 255 answers, and 201 of the participants completed the survey. Among the participants who completed the survey, 134(66.67\%) were from the United States.

\subsection{Procedure}

The references for the survey design are the Flow for Present Questionnaire (FPQ) and the Flow State Scale (FSS). This research designed a survey containing demographic questions (age), Likert scales that measure the self-reported levels of flow conditions, concentration, involvement, and enjoyment. And there are open-ended questions that the respondents can describe their feelings more specifically. This study is looking forward to collecting both quantitative and qualitative answers from the respondents to better understand the research question. After answers are collected, researchers categorize and code the open-ended questions' answers, making them quantitative; and use them as qualitative data to help this research gain more information about respondents' feelings. Collected surveys with an answering time of fewer than 100 seconds, same answers for all questions, and incomplete answers are defined as invalid responses and were filtered out. Since the study focuses on U.S. undergraduate students aged from 18 to 24, participants outside of the U.S. are filtered out. Among 201 respondents who completed the survey, $134(66.67 \%)$ were from the United States. After filtering the answers according to the principle mentioned before, 96 answers to the questions for video games are valid, and 93 answers to the questions for TV shows are valid.

\subsection{Data Analysis}

From the data collected from the survey, the analysis takes the type of entertainment activities as the categorical independent variable and their self-reported levels of flow conditions, concentration, involvement, and enjoyment as numerical dependent variables. Answers in each group are independent and can not influence answers in each other. Moreover, the sample of the data from the population is randomly selected. We will conduct Welch's t-tests for matching independent and dependent variables and finding the correlation between each pair. The null hypothesis is that the mean of the self-reported levels of flow conditions, concentration, involvement, and enjoyment has no difference in the video game group and TV show group. The alternative hypothesis is that the mean of the selfreported levels of flow conditions, concentration, involvement, and enjoyment in the video game group is greater than that in the TV show group. Moreover, a 95\% confidence interval is used. To decrease the likelihood of Type I error, the analysis uses a more stringent alpha level of .01.

\section{RESULT}

Assuming a normal distributed population distribution, this study conducted an independent sample t-test to test the hypothesis that the mean of self-reported levels of flow scale is greater in the video game group than in the TV shows group. The sample evidence suggests that the mean of the flow condition scale for video games experiences $(\mathrm{M}=3.79, \mathrm{SD}=0.79)$ is highly significantly greater than the mean for TV show experiences $(\mathrm{M}=3.35, \mathrm{SD}=1.10) ; \mathrm{t}(670)=6.24, \mathrm{p}<$ .001 . The sample evidence also shows that differences between the mean of the self-reported levels of concentration for video games experiences $(M=3.80$, $\mathrm{SD}=0.93)$ and the mean for TV show experiences $(\mathrm{M}=$ $3.85, \mathrm{SD}=0.91)$ is not significant; $\mathrm{t}(376)=-0.49, \mathrm{p}=$ 0.69 . And the data evidence illustrate that differences between the mean of the self-reported levels of involvement for video games experiences $(\mathrm{M}=3.70, \mathrm{SD}$ $=0.89)$ and the mean for TV show experiences $(\mathrm{M}=$ $3.62, \mathrm{SD}=0.98)$ is not significant; $\mathrm{t}(556)=1.03, \mathrm{p}=$ 0.15. Moreover, the data evidence implies that differences between the mean of the self-reported levels of enjoyment for video games experiences $(\mathrm{M}=4.02, \mathrm{SD}$ $=0.72)$ and the mean for TV show experiences $(\mathrm{M}=$ $3.97, \mathrm{SD}=0.85)$ is not significant; $\mathrm{t}(361)=0.59, \mathrm{p}=$ 0.28 . Thus, this result has shown sufficient evidence to reject the null hypothesis for the flow conditions. There is no significant evidence to reject the null hypothesis for the three dimensions of the flow state scale.

For the open-ended question, there is an interesting difference between the two groups. In respondents' description of their video game experience, the majority $(43.47 \%)$ of participants used words such as "happy" or "fun"; and, in their descriptions of their experiences of watching TV shows, the majority $(63.01 \%)$ used words such as "enjoy" and "relax". Other words that occur in responses for video games are "engaging", "engrossed", 
"exciting", and "satisfied". In response to TV shows, the words occurring are "entertained", "escape", and "interesting". Also, three responses were mentioning that they are "addicted" to video games. And three responses are mentioning that they take TV shows as a secondary activity. Some respondents mentioned in open-ended questions that they were focused on the TV shows because of the content, character and storyline. Moreover, some participants mentioned that while watching TV shows, they were "passively entertained."

\section{DISCUSSION}

\subsection{Significant Difference in Flow Condition}

The data evidence has shown a highly significant difference in the levels of self-reported flow condition between experiences of video games and TV shows. It implies the video game possesses a balance between challenge and skill, immediate feedback and a clear goal since it scores higher in scales of flow condition. This result is consistent with previous studies and flow theories [1], which takes video games as examples of flow activities. In the field of entertainment, whether flow conditions exist or not may not have a great influence on levels of enjoyment; however, it may affect how the audience or players were entertained. According to the collected answers to the open-ended questions, responses that described the experience of watching TV shows mentioned that they were "passively entertained." This implies that entertainment activities that possess a low flow condition scale, such as watching TV shows, is likely to be passive entertainment. Future research may focus on whether flow condition exists or not correlated with whether the entertainment activity is passive or active.

\subsection{No significant difference in Flow Experience}

Compared with the significant results discovered in the context of flow conditions, there's no significant distinction on the level of flow scale (scoring) between TV shows and Video games. Based on the analysis of the open-ended question in the survey and previous studies conducted by researchers, an individual's ability to focus on the TV increase when the length of the online videos is shorter compared to the longer ones [9]. With that being said, the time viewers need to spend on different genres of TV shows contributes to the level of attention span, such as comedy compared to documentary. As a result indicated, participants who feel "escaped" and "focused" tend to score higher in questions associated with concentration and involvement by watching sitcoms or animated series, usually episodes that last between 3545 minutes. Involvement is the kind of feeling that the participants say: "that was fun, "or "that was enjoyable. " People tend to experience it as a unified flowing from one moment to the next, so basically, we feel in control of our actions, and there is little distinction between self and environment.

According to our findings, people are generally capable of a longer involvement span when they do something that they find enjoyable or intrinsically motivating. The level of involvement is also increased if the person is able to perform the task fluently. In addition, hunger, noise, and emotional stress can reduce the time and immersion that someone focuses on the task. Other factors that explain the relatively high attention span score on TV shows are that commercial and Television companies used cognitive dissonance and storytelling techniques. Auditory-visual dissonances, for example, are the sound and image effect that combine as one stimulus to create uncomfortable feelings from the viewer and keep them guessing [10]. The producer usually reveals a decent amount of information instead of the whole ending or point to create a maximum effect in the climax.

Besides, it's hard for viewers to stay focused in film and TV shows, given there are advertisements between each episode and possible distractions from the outside environment. To solve that issue, producers utilized wonderful storytelling tricks by analyzing flow state to keep the viewer's eyes on the content they wanted. The concept of "flow" in television studies refers to the scheduling of programs, and the advertisement breaks inside and between them as a continuum. The ultimate product is designed to keep the viewer focused on a single station. Aside from that, it's impossible to narrow down the specific meaning of the term. Critics also use flow to the experience of the viewer, who supposedly views the pauses in the television schedule as part of the program rather than an interruption [11]. In short, the structure of the entire TV series is a single-episode type planned flow, and the purpose is to concentrate the viewer's attention in one short episode. Besides, there will always be exciting connections or related plots that connect the two episodes together. The plotlines in TV shows compare to the film are relatively simple. One or two clear plotlines can assist the viewers in understanding what they're watching, which creates a sense of ease and relaxation [11]. Participants who reported "relax" and "enjoy" in the open-ended questions tend to choose soup drama and streaming TV, proving that simplicity creates relaxation.

To conclude, video length and attention span theory, sensory stimuli, and storytelling are all alternative explanations to examine that participants' scores on the flow scale are insignificance between video game playing and watching tv shows. 


\section{CONCLUSION}

The significant difference of self-reported level of flow condition between video games and TV shows implies entertainment activities, such as video games, in which participants face the right amount of challenge, have immediate feedback and a clear goal, can score higher in flow condition. Also, there is no significant difference in flow experience (concentration, involvement, and enjoyment) between video games and TV shows. It seems that the flow condition barely influences entertainment activities. Considering the characteristic of entertainment, those activities can entertain individuals in both passive ways and active ways. Activities with flow condition, such as video games, is likely to be active entertainment that required participants to take an active part in it. And TV shows, which does not need the audience to take actions to involve, is a kind of passive entertainment. This difference can be an alternative explanation of the results. Moreover, TV shows that contain interesting content, exciting storyline, and sufficient new knowledge can possibly promote the levels of flow state scale in a different way than flow conditions do.

\section{AUTHOR'S CONTRIBUTION}

Celestia Gao contributed to the literature review. Zihan $\mathrm{Lu}$ contributed to the methodology and data analysis. Both authors contributed to the background research, survey design, and discussion.

\section{REFERENCES}

[1] Csikszentmihalyi, M. (2014). Play and intrinsic rewards. In Flow and the foundations of positive psychology (pp. 135-153). Springer, Dordrecht.

[2] Wright, Jancee (2011) "The effects of video game play on academic performance," Modern Psychological Studies: Vol. 17 : No. 1, Article 6.

[3] Williams, Raymond. Television: Technology and cultural form. Routledge, 2004. https://doi.org/10.4324/9780203426647

[4] Cowley, B., Charles, D., Black, M., \& Hickey, R. (2008). Toward an understanding of flow in video games. Computers in Entertainment (CIE), 6(2), 1-27. https://doi.org/10.1145/1371216.1371223

[5] Jackson, S., \& Eklund, R. (2002). Assessing flow in physical activity: The flow state scale-2 and dispositional flow scale-2. Journal of Sport and Exercise Psychology, 24, 133-150.

[6] Jackson, S. \& Marsh, H. (1996). Development and validation of a scale to measure optimal experience: The Flow State Scale. Journal of Sport \& Exercise Psychology, 18, 17-35.
[7] Wang, C.K.J., Liu, W.C. \& Khoo, A. The Psychometric Properties of Dispositional Flow Scale-2 in Internet Gaming. Curr Psychol 28, 194201 (2009). https://doi.org/10.1007/s12144-0099058-X

[8] Csikszentmihalyi, M., \& Csikzentmihaly, M. (1990). Flow: The psychology of optimal experience (Vol. 1990). New York: Harper \& Row.

[9] Geri, N., Winer, A., \& Zaks, B. (2017). Challenging the six-minute myth of online video LECTURES: CAN INTERACTIVITY expand the attention span of learners? Online Journal of Applied Knowledge Management, $\quad 5(1)$, 101-111. https://doi.org/10.36965/ojakm.2017.5(1)101-111

[10] GRIMES, T. O. M. (1991). Mild Auditory-Visual dissonance in television news may Exceed viewer Attentional Capacity. Human Communication Research, 18(2), 268-298. https://doi.org/10.1111/j.1468-2958.1991.tb00546.x

[11] Thompson, K. (2003). Storytelling in film and television. Harvard University Press. 